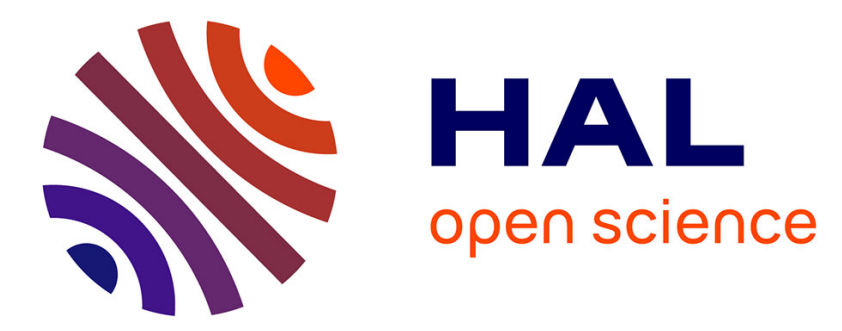

\title{
Intracellular parasitism, the driving force of evolution of Legionella pneumophila and the genus Legionella
}

\author{
Laure Gomez-Valero, Carmen Buchrieser
}

\section{To cite this version:}

Laure Gomez-Valero, Carmen Buchrieser. Intracellular parasitism, the driving force of evolution of Legionella pneumophila and the genus Legionella. Microbes and Infection, 2019, 21 (5-6), pp.230-236. 10.1016/j.micinf.2019.06.012 . pasteur-02895319

\section{HAL Id: pasteur-02895319}

\section{https://hal-pasteur.archives-ouvertes.fr/pasteur-02895319}

Submitted on 9 Jul 2020

HAL is a multi-disciplinary open access archive for the deposit and dissemination of scientific research documents, whether they are published or not. The documents may come from teaching and research institutions in France or abroad, or from public or private research centers.
L'archive ouverte pluridisciplinaire HAL, est destinée au dépôt et à la diffusion de documents scientifiques de niveau recherche, publiés ou non, émanant des établissements d'enseignement et de recherche français ou étrangers, des laboratoires publics ou privés.

\section{(1)(1) $\$(0)$}

Distributed under a Creative Commons Attribution - NonCommercial - ShareAlikel 4.0 
1 Intracellular parasitism, the driving force of evolution of Legionella

3

4

5

6

7

8

9

10

11

12

13

14

15

16

17

18

19

20

21

22

23

24

25

26

27

28

29

30

31

32

33

34

35

36

37

\section{pneumophila and the genus Legionella}

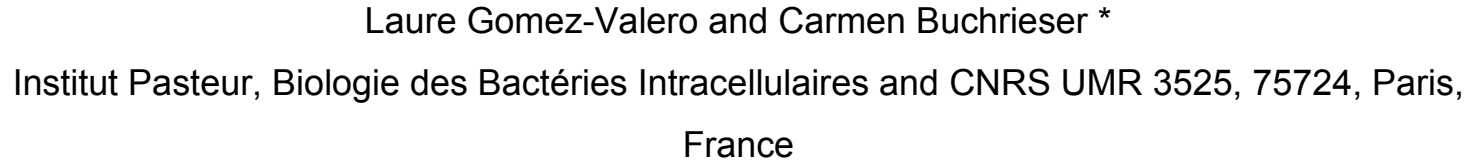

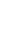

(1)

11

12

13

5

\section{Running title: Co-evolution of Legionella and protozoa}

(1)

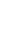

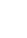

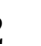

3

*For correspondence:

(

Carmen Buchrieser

Institut Pasteur

Biologie des Bactéries Intracellulaires

28, rue du Dr. Roux, 75724 Paris Cedex 15, France

Tel: (33-1)-45-68-83-72

E-mail:cbuch@pasteur.fr 
Abstract

Legionella pneumophila is an intracellular pathogen that causes a severe pneumonia called Legionnaires' disease that is often fatal when not promptly diagnosed and treated. However, L. pneumophila is mainly an environmental pathogen of protozoa. This bacterium parasitizes free-living amoeba and other aquatic protozoa with which it co-evolved over an evolutionary long time. Due to the close relationship between hosts and pathogens, their co-evolution

44 leads to molecular interactions such as the exchange of genetic material through horizontal gene transfer (HGT). Those genes that confer an advantage to the bacteria were fixed in their genomes and help these pathogens to subvert host functions to their advantage. Genome sequencing of $L$. pneumophila and recently of the entire genus Legionella that comprises over 60 species revealed that Legionellae have co-opted genes and thus cellular functions from their eukaryotic hosts to a surprisingly high extent never observed before for an prokaryotic organism. Acquisition and loss of these eukaryotic-like genes and eukaryotic domains is an on-going process underlining the highly dynamic nature of the Legionella genomes. Although the large amount and diversity of HGT that occurred between Legionella and their protozoan hosts seems to be unique in the prokaryotic world the analyses of more and more genomes from environmental organisms and symbionts of amoeba revealed that such genetic exchanges occur among all amoeba associated bacteria and also among the different microorganisms that infect amoeba such as viruses. This dynamic reshuffling and gene-acquisition has led to the emergence of major human pathogens such as Legionella and may lead to the emergence of new human pathogens from the environment. 
Pathogenicity refers to the ability of an organism to cause disease and to harm the host. The more virulent a pathogen the higher the degree of host damage it can induce, but virulence evolves to the level that optimizes the pathogens reproduction and transmission rate. Pathogenicity and virulence developed through co-evolution of pathogens with its hosts, a major driver of evolution and biological innovation over millions of years. Host-pathogen coevolution is very widespread across ecosystems, but perhaps the best studied is that occurring between plants, animals or humans and pathogenic parasites, fungi, viruses or bacteria. The result of this reciprocal selection lead to the evolution of sophisticated mechanisms to subvert host functions and shaped the immune defences in eukaryotic cells that should eliminate invading microorganisms.

Due to the close relationship between hosts and pathogens, their co-evolution leads to molecular interactions such as the exchange of genetic material through horizontal gene transfer (HGT). During evolution the sequences acquired can be adapted to the recipients species and thereby improve its fitness and affect the interaction between the pathogen and its host. Inter-bacterial HGT was first described in 1959 when the ability of Shigella to incorporate drug resistance genes from other Shigella strains and from Escherichia coli was discovered (1). Since then, it became clear that HGT is an important force driving the evolution of bacteria and archaea, as well as that of unicellular eukaryotes (2). It has now also been shown that prokaryotes cannot only exchange genetic material with other prokaryotes and viruses with viruses, but also between them and with eukaryotes. However, there are only few reports of eukaryote-to-prokaryote HGT.

83 One intriguing case where eukaryote-to-prokaryote HGT has been described is the co-evolution of Legionella with protozoa. Legionella are environmental bacteria belonging to the class of $\gamma$-proteobacteria. The genus contains over 60 species, among which Legionella pneumophila and Legionella longbeachae are major human pathogens that are known as the etiological agent of Legionnaires' disease, a severe pneumonia that is often fatal when not treated promptly $(3,4)$. Legionella are ubiquitous in fresh water reservoirs worldwide but certain species are also found in moist soil, where they parasitize within free-living protozoa (5). The finding that these bacteria replicate intracellularly in environmental protozoa such as Acanthamoeba castellanii, Verbamoeba veriformis or Hartmanella veriformis led to a new perception in microbiology: the ability of a bacterium to replicate within human monocytes and alveolar macrophages, may be derived from the conserved cell biology between amoeba, its natural host in aquatic environments, and human phagocytic cells (5-7). 
Indeed, L. pneumophila encodes a type IV secretion system named Dot/lcm $(8,9)$ that is secreting proteins, which allow this bacterium to manipulate host functions in protozoan and in human cells. Furthermore, several of the traits that contribute to the fitness of L. pneumophila in the environment (protozoa) also facilitate its growth in alveolar macrophages (reviewed in $(4,10-12)$ ). However, how the adaptation of Legionella to eukaryotic cells and the ability to replicate intracellularly may have evolved on the molecular level was not known.

The L. pneumophila genome sequence, a breakthrough in the understanding of Legionella-protozoa co-evolution

Legionella pneumophila was one of the human pathogens whose genome was completely sequenced only in 2004 . The analysis of its genome sequence was key for a new understanding of the strategies employed by Legionella to subvert host functions as the genome sequence uncovered an intriguing feature of the L. pneumophila genome. It encodes an unmatched large number and diversity of bacterial proteins with eukaryotic-like properties (13). Among the about 3000 protein coding genes predicted in the genome, more than 150 proteins with high similarity to eukaryotic proteins or carrying eukaryotic motifs were predicted, representing about $5 \%$ of its protein-coding capacity, a number that increased later when systematic searches were employed. Examples of protein domains that had been identified in the L. pneumophila genome are F-box and U-box domain proteins, SETdomains, Sel1-domains, STPK domains and Ankyrin domains (13). Examples for proteins homologue to eukaryotic proteins, which are proteins with $\geq 30 \%$ amino acid similarity over at least two thirds of the eukaryotic protein length are a eukaryotic glycoamylase, apyrases, or a sphingosine-1 phosphate lyase. This finding led to the suggestion that L. pneumophila secretes these proteins in the host cell to subvert eukaryotic signalling pathways by mimicking host cell functions (13). Indeed, also the first described Dot/lcm effector RalF that was identified before the genome was sequenced, encodes a eukaryotic Sec7 domain. Sec7 domains are components of Arf-specific guanine nucleotide exchange factors (GEFs). GEFs catalyse the nucleotide exchange of Arfs thereby converting them from an inactive state (GDP-bound) to the active one (GTP-bound). Like in a eukaryotic cell, following secretion into the host cell, RalF recruits Arf-1 and then functions like an Arf-1 specific GEF (14).

Based on the information gleaned from the genome sequence analyses many of the eukaryotic-like effectors were functionally analysed to learn whether they are bacterial weapons employed to subvert host functions as predicted. Indeed, each of the effectors analysed to date, encoded the predicted eukaryotic function and was shown to be part of a sophisticated effector network that evolved to manipulate the host cell. These effectors modulate a plethora of host cell processes including vesicular trafficking, apoptosis, 
133 autophagy, protein synthesis, ubiquitination, epigenetic modifications, and induce many 134 different post translational modifications (PTM) (15). They may induce the PTMs directly as 135 do AnkB or LubX that contain an F-box or U-box motif, respectively and function as E-3 136 ligases that transfer ubiquitin moieties to host proteins (15-17) or they may recruit host 137 enzymes such as the eukaryotic protein prenyl transferases to achieve membrane 138 localization of the respective effector $(18,19)$.

139 More than 330 effectors secreted by the Dot/Icm type IV secretion system (T4SS) and 140 over 25 proteins secreted by the type II secretion system (T2SS) have been described for

141 L. pneumpohila (20-22). With a genome size of in average $3.2 \mathrm{Mb}$ and 3100 protein coding 142 genes this astonishing number of over 350 secreted proteins which represent over $10 \%$ of 143 the L. pneumophila proteome is not matched by any other known bacterial pathogen. The 144 closest comes Coxiella burnetti, which has a genome size of about $2 \mathrm{Mb}$ and about 2100 145 predicted protein coding genes $(21,22)$ and over 100 secreted effector proteins (23). Thus 146 the question arises why does Legionella need that many effector proteins? This question 147 becomes even more puzzling as many of the effectors studied to date do not show any or at 148 least no strong intracellular growth defect when deleted nor does even the simultaneous 149 deletion of over 60 effectors obtained through large chromosomal deletions that carry these 150 genes (24). Based on the different data, it is thought that $L$. pneumophila encodes such a 151 high number of secreted proteins, to fine-tune the host pathogen interactions to allow the 152 replication in many different protozoan hosts. Thus the redundancy of effector functions 153 observed in intracellular growth in human or mouse macrophages might be beneficial for 154 Legionella when parasitizing protozoa in the environment as L. pneumophila may use 155 different effector sets adapted to different protozoan species.

157 The genus Legionella co-opts eukaryotic functions to an unprecedented high number 158 and diversity

159 Legionella pneumophila is part of a large genus of over 65 species of which most are 160 harmless, environmental bacteria found in aquatic environments associated with amoeba. 161 Legionella longbeachae, is the second species often found in human disease as it is a 162 frequent cause of Legionnaires' disease in Australia, New Zealand and Southeast Asia but it 163 emerges lately also in Europe and the United States (25). However most of the other 164 Legionella species have been only rarely or never found in human disease and only little is 165 known about them. Thus an exciting question to answer was, whether the presence of 166 eukaryotic genes and eukaryotic domains is a general feature of the Legionella genomes. A 167 first answer came from the analyses of the $L$. longbeachae genome, as indeed the effector 168 repertoire seemed of similar size and a high number of eukaryotic domains and proteins had 169 been identified (26). However the surprising finding was, that only about $34 \%$ of the 
170 L. pneumophila effectors were conserved in the L. longbeachae genome, but 51 new,

171 putative Dot/lcm substrates specific for $L$. longbeachae that encode eukaryotic-like domains

172 were identified (26). Related to a different life style, $L$ longbeachae is found in moist soil and

173 potting soil, genes that might have been acquired from plants have been identified, such as

174 proteins with pentatricopeptide repeat (PPR) domains, a family of proteins that is greatly

175 expanded in plants.

176 Recently the nearly entire genus Legionella has been sequenced and analysed (27,

177 28). This disclosed a fascinating and unique feature of these bacteria. A highly dynamic and

178 diverse effector repertoire of over 18000 proteins that contain at least 137 different

179 eukaryotic domains and over 200 different eukaryotic proteins was discovered (28).

180 Comparative genome and evolutionary analyses brought evidence that Legionella species

181 have acquired these eukaryotic-like proteins from all domains of life, plant, animal, fungal,

182 and archaea (28). A particular exciting finding was the identification of 184 genes that are

183 predicted to encode small GTPases, 71 of which are Rab GTPases. All have the best Blast

184 hit with proteins form protozoan organisms such as Entamoeba or Tetrahymena.

185 Furthermore phylogenetic analyses indicate that these proteins are indeed acquired from

186 protozoan hosts (28). Thus RabGTPases are a unique feature of the genus Legionella.

187 Most interestingly, despite the enormous diversity of eukaryotic domains present in

188 the Legionella effectors, it seems that certain signalling pathways are exploited by all

189 species. Indeed, quasi all genomes contain U- and/or F-box proteins suggesting that the

190 exploitation of ubiquitin signaling is of outmost importance to succeed replication inside

191 eukaryotic host cells (28). Another example is the eukaryotic-type ecto-NTPDases

192 (apyrases), which are conserved in all species analysed. It has been shown that this protein

193 confers to L. pneumophila the ability to hydrolyse ATP, a function that seems necessary for

194 optimal intracellular replication (29). Recently the structure of NTPDases from a legume plant

195 revealed that these NTPDases could adopt two conformations depending on the molecule

196 and co-factor bound in the active site (30). Interestingly this phenomenon had been

197 previously described in Rattus norvegicus, Toxoplasma gondii NTPDaselll and the

198 L. pneumophila NTPDasel suggesting a common catalytic mechanism across the domains of

199 life. This structural similarity again supports the idea that Legionella have acquired these

200 functions from eukaryotic organisms. Thaumatin domains that are considered a prototype for

201 a pathogen-response protein domain in fungi, plants, and animals are also present in all

202 Legionella genomes (28). Another interesting domain is the SET domain encoded by RomA

203 of L. pneumophila where it has been shown to induce a unique host chromatin modification

204 (31). This domain is present in nearly all Legionella species but L. longbeachae (28)

205 suggesting that modification of histones is an important mechanism by which Legionella

206 facilitate their intracellular survival. Thus although most surprisingly only a set of 8 conserved 
core effectors was identified in the genus Legionella $(27,28)$ the identification of the

208 presence of conserved domains suggests that one could perhaps define a core set of 209 eukaryotic signalling pathways that intracellular bacteria need to modulate to replicate 210 intracellularly.

\section{Inter-domain horizontal gene transfer and the emergence of a human pathogen}

213 The high number and wide variety of eukaryotic functions discovered in the Legionella 214 genomes suggested that inter domain horizontal gene transfer may be the mechanism of 215 acquisition and that these proteins and domains of eukaryotic origin witness the tight co216 evolution between Legionella and its protozoan hosts $(13,26,32-34)$. Many reviews on the 217 functions of these different effectors of L. pneumophila and how they subvert host signalling 218 pathways have been published in the last years (e.g. $(15,35-40))$ thus we will here further 219 detail only one "eukaryotic-like" effector protein, the L. pneumophila sphingosine-1 220 phosphate lyase named $L p S p l$, as this protein is an excellent example of how these 221 eukaryotic like proteins might have been acquired and evolved.

222 Sphingolipids are major components of all eukaryotic cellular membranes. They have 223 important functions as signalling molecules in the eukaryotic cell by regulating processes 224 such as the stress response, cell proliferation, apoptosis, angiogenesis, genetic diseases, 225 and resistance to chemotherapy (41). Simplified, sphingomyelin, present in plasma 226 membranes is hydrolysed by sphingomyelinase to ceramide that can also be de novo 227 synthetized, which then is converted by ceramidase to sphingosine, which is phosphorylated 228 by a sphingosine kinase to sphingosine-1 phosphate that can be converted by sphingosine-1 229 phosphate lyase (Spl) to hexadecanal + Ethanolamine-P (42). Interestingly, sphingolipid 230 biosynthesis was shown to be conserved in Acanthamoeba castellanii and appears to be 231 generally conserved among protozoa (43). Only very few bacteria such as 232 Sphingobacterium, Sphingomonas and Bacteroides and Bdellovibrio stolpii are able to 233 synthetize sphingolipids (42). Thus it was an intriguing finding that the L. pneumophila 234 genome encodes several eukaryotic enzymes participating in the sphingolipid pathway, such 235 as sphingosine kinase, sphingomyelinase and sphingosine-1 phosphate lyase (44).

236 The L. pneumophila sphingosine-1 phosphate lyase named LpSpl, was further 237 characterized. Its structural analyses showed that $L p S p l$ has a dimeric multidomain 238 architecture that is very similar to the previously characterized SPL structures of the human 239 (hSPL) and the yeast (Dpl1p) enzyme. Their comparison revealed that the active site of the 240 enzyme was conserved among the LpSpl and hSpl and activity analyses confirmed that the 241 L. pneumophila Spl shows indeed sphingosine-1 phosphate lyase activity like its human 242 counterpart (45). Furthermore metabolomics analyses of L. pneumophila infected human 243 macrophages revealed that $L$. pneumophila $L p S p$ targets the sphingolipid metabolism of the 
244 host cell directly to modulate the levels of sphingosine and restrains autophagy (45). Thus,

$245 L p S p l$ is an enzyme that modulates the host cell sphingolipid metabolism to the pathogens 246 advantage.

247 The question arises "what is the origin of such an eukaryotic enzyme in an prokaryotic 248 genome?". To answer this question we have undertaken phylogenetic analyses of this gene 249 by recruiting homologous sequences from a database containing only completed genome 250 sequences. Selected representatives of all eukaryotic groups and one representative of each 251 bacterial species were included in the analyses. After Blastp only significant hits (e-value $<10$ $252 \times 10^{-4}$ ) were retained, and only one hit for each species was included in the analysis. The 253 resulting phylogenetic tree is shown in Figure 1A. Indeed, the $L$. pneumophila $L p S p l$ gene is 254 embedded in the same clade as the eukaryotic sequences from Entamoeba spp., 255 Tetrahymena thermophila and Paramecium tetraurelia Spl, which is suggesting that $L p S p l$ 256 was acquired by horizontal gene transfer from a protist host as also suggested earlier (46, 257 47). The analyses of the distribution of the sphingosine-1 phosphate lyase in the genus 258 Legionella reveals that this enzyme is present in 16 of the 58 Legionella species/subspecies 259 analyzed (Figure 1B) suggesting that the remaining 42 species have evolved other ways to 260 manipulate the host sphingolipid metabolism or employ different strategies to restrain 261 autophagy. Indeed, even among different L. pneumophila species are differences in how 262 they subvert the autophagy pathway. An example is RavZ, an effector of L. pneumophila 263 strain Philadelphia that inhibits autophagosome maturation through irreversible ATG8 264 decongugation that is absent from strain Paris (48).

265 To better understand the evolutionary history of the sphingosine-1 phosphate lyase $266(s p /)$ gene in the genus Legionella, we have analysed the phylogenetic relationship of the 16 267 Legionella spl genes. As shown in Figure 2A, the protein similarity ranges from 63-100\% 268 and five highly related groups can be distinguished. L. pneumophila subsp pneumophila, 269 L. pneumophila subsp pascuelleii, L. pneumophila subsp fraserii and L. waltersii form one 270 group where the Spl sequence shows $95-100 \%$ similarity to the L. pneumophila LpSpl 271 sequence. A second group with $70 \%$ sequence similarity is formed by $L$. gresiliensis and 272 L. busanensis, a third group that shows $67-69 \%$ similarity to $L p S p l$ contains the species 273 L. hackeliae, L. jamestowniensis and L. brunensis and finally the least conserved group 274 contains five species that show $63-68 \%$ sequence similarity to $L p S p l$ (Figure 2A). Thus the 275 phylogeny of the different Spl proteins in the genus Legionella, suggests either acquisition of 276 an spl gene in a common ancestor and subsequent diversifying evolution and losses in many 277 species or multiple acquisitions. To answer this question, we overlapped the distribution of 278 the $s p /$ sequences on the phylogeny of the genus (Figure 2B) and carried out evolutionary 279 analysis of presence/absence using GLOOME and stochastic mapping. These analyses 280 showed that the $s p /$ gene has been acquired at least four times during the evolution of the 
281 genus (green arrows) and has also been lost several times (red dots). Thus gene gain and

282 loss seems to be an on-going process that shapes the Legionella genomes.

283 L. pneumophila was one of the first examples for evidence of eukaryote to prokaryote

284 gene transfer. However, genome analyses from environmental bacteria including symbionts

285 of amoeba showed that eukaryotic domains were also present in the amoeba symbiont

286 Amoebophilus asiaticus (49). An analyses of 480 genomes of different prokaryotes revealed

287 that eukaryotic domains are significantly enriched in the genomes of many amoeba-

288 associated bacteria such as Chlamydiae, Rickettsia bellii, Francisella tularensis, or

289 Mycobacterium avium (49). This indicates that phylogenetically and ecologically diverse

290 bacteria, which thrive inside amoebae, exploit common mechanisms for interaction with their

291 hosts and are all exchanges genetic material (49). Recently it was also proposed that

292 amoeba-fungal interaction might select for traits that promote survival during animal infection

293 and thereby contribute to virulence (50). Thus similar processes may contribute to the

294 evolution of other amoeba-associated bacteria and fungi and may lead to the emergence of

295 new human pathogens.

296

297 Horizontal gene transfer among amoeba associated bacteria or viruses within amoeba

298 The availability and comparison of genome sequences from organisms belonging to all 299 domains of life and residing in different environmental niches brought evidence that HGT 300 may occur between many organisms and not only between closely related species but even 301 between different domains of life. In this context amoeba seem to be a privileged 302 environment for DNA exchange. Indeed, Legionella seems to have exchanged genetic 303 material also with viruses that infect amoeba, as it was reported that L. pneumophila 304 encodes proteins homologous to proteins found in the mimivirus genome (51), a virus that 305 infects Acanthamoeba $(34,52)$. Most interestingly, this situation seem to be reciprocal as 306 intracellular bacteria appear to have transferred genes also to the mimiviral genome, some of 307 which are involved in the parasitic adaptations of the mimivirus (52).

308 In addition to gene exchange between amoeba-associated bacteria such as 309 Legionella with viruses, there is also evidence of gene exchange between different bacteria 310 infecting amoeba. Rickettsia which also replicate in amoeba, contain genes encoding a 311 putative conjugal DNA transfer system highly similar to that of Protochlamydia amoebophila 312 UWE25, an obligate symbiont of amoebae and other genes highly similar to homologues in 313 intracellular bacteria of amoebae (53). Indeed, one of the secreted effectors of 314 L. pnemophila, RalF contains a eukaryotic Sec-7 domain (14). The analyses of the 315 evolutionary history of this domain reveals that a similar domain is present in the Rickettsia 316 genomes, and that both, Rickettsia and Legionella Sec-7 sequences are embedded within 317 eukaryotic sequences suggesting that one of the bacteria acquired this domain from an 
318 amoeba host and then the bacteria exchanged this domain among them (54). Another

319 interesting report reveals that amoeba may also acquire genes from their bacterial parasites

320 or symbionts. The anaerobic protist Mastigamoeba balamuthi encodes p-cresol- and indole-

321 producing enzymes that most likely originated from phagocytized bacteria in the protist's

322 anoxic habitat and allowed the eukaryotic recipient to produce the bacterial weapon $p$-cresol

323 at bacteriostatic concentrations (55).

324 Thus gene exchange between many different organisms may take place and if the 325 acquired DNA confers an advantage to the recipient, it will be fixed and will evolve further 326 with the new genome. Thus evolutionary analyses might miss the real extent of these gene 327 exchanges as there are likely genes e.g. in prokaryotes that originated from eukaryotic 328 species but there are no identifiable eukaryotic homologs presumably due to substantial 329 evolution of these proteins after their acquisition by the bacteria as suggested for the 330 Legionella SH2 domain proteins (56). Another reason that makes it difficult to trace the 331 evolutionary history of certain genes is due to the fact that we do not have enough 332 sequencing data for environmental protozoa, fungi and bacteria. Once databases are 333 enriched with such sequences we might see even more genetic exchange than thought.

\section{Conclusion}

336 Amoeba associated bacteria seem to strive in an environment that is prone to HGT. Gene 337 exchange among amoeba associated bacteria such as Legionella, Chlamydia or Rickettsia 338 as well as between the amoebal host and the parasitizing bacteria or the viruses present in 339 amoeba takes place $(13,26,34,47,57-61)$. The investigation of the function of these 340 horizontally acquired genes, suggests that they confer a selective advantage to the bacteria. 341 Indeed, Legionella have transformed these proteins, using them as "tools of oppression" to 342 hijack host cellular functions, in particular targeting signal transduction, protein turnover and 343 chromatin modifying pathways. However, the finding that Legionella species have acquired 344 eukaryotic-like proteins from all domains of life, plants, animals, fungi, and archaea, in an 345 unprecedented high number and large diversity opens many new questions. One intriguing 346 question is "what is the mechanism by which these transfers occur?" and "how is the foreign 347 DNA integrated in the prokaryotic genomes?". An interesting finding that may be related to 348 the inter domain gene transfer is the identification of a gene predicted to encode a group II 349 intron reverse transcriptase in the L. pneumophila genome. Thus a possibility is that 350 L. pneumophila incorporates also RNA from its host, a fact that would explain why the 351 eukaryotic genes in Legionella do not carry introns. The proof that RNA may be transferred 352 horizontally would be the discovery of a new key mechanism for evolution and adaption of 353 bacteria. Furthermore Legionella are able to develop competence for natural transformation 354 (62), a major mechanism of HGT which may act in the intracellular environment of amoeba. 
355 However, experimental proof is missing yet. Thus many exciting questions on the evolution 356 of Legionella that may teach us also how new human pathogens may evolve from the 357 environment remain to be answered. The knowledge on these evolutionary processes will be 358 a precious help to avoid the emergence of new pathogens and gives an exciting outlook on 359 future research.

360

361 Acknowledgements

362 Work in the CB laboratory is financed by the Institut Pasteur and has received funding from 363 the French Government's Investissement d'Avenir program, Laboratoire d'Excellence 364 "Integrative Biology of Emerging Infectious Diseases" (grant $n^{\circ}$ ANR-10-LABX-62-IBEID) and 365 grant ANR 15 CE17 001403 
Figure 1: The sphingosine -1 phosphate lyase gene was acquired by horizontal gene transfer from protozoa but is not conserved throughout the genus. A) Phylogeny of the sphingosine-1 phosphate lyse (Lpp2128) of L. pneumophila strain Paris and homologous sequences from eukaryotic and prokaryotic organisms. Homologous sequences were recruited from a database containing only completed genome sequences. Selected representatives of all eukaryotic groups and one representative of each bacterial species are

375 (e-value $<10 \times 10^{-4}$ ), and only one hit for each species was retained. The alignment was 376 performed with Muscle for Lpp2128, and followed by manual curation. The phylogeny was 377 reconstructed using a distance method (NJ) with 1000 bootstrap replicates. The 378 corresponding support values are shown in each node (values lower than 50 are not 379 represented). Bars represent $20 \%$ and $10 \%$ of estimated phylogenetic divergence, 380 respectively. B) Distribution of the orthologous genes of the sphingosine-1 phosphate lyse 381 Lpp2128 in the genus Legionella. Orthology prediction has been done using the Pan 382 Genome Ortholog Clustering Tool (PanOCT) with the following parameters: amino acid 383 percentage identity cut-off $30 \%$, BLAST e-value cutoff $10^{-5}$, and minimum percentage match 384 length of subject and query $65 \%$

Figure 2: The sphingosine-1 phosphate lyase genes have been acquired and lost multiple times during the evolution of the genus Legionella. A) Phylogeny of the sphingosine-1 phosphate lyse (Lpp2128) of L. pneumophila strain Paris and the corresponding orthologous proteins in other Legionella species. The tree was constructed by likelihood using the software RAxML with 100 bootstrap replicates. The corresponding support values are shown in each node. B) Gain/loss prediction for the sphingosine-1 phosphate lyase in the different Legionella species. The prediction was done based on parsimony using the program GLOOME with double cost of gain versus loss. Green arrows represent acquisition events and red dots on the branches represent gain events 
397

398

399

400

401

402

403

404

405

406

407

408

409

410

411

412

413

414

415

416

417

418

419

420

421

422

423

424

425

426

427

428

429

430

431

432

433

434

435

436

437

438

439

440

441

442

443

444

445

446

447

1. Ochiai K, Yamanaka T, Kimura K, Sawada O. Inheritance of drug resistance (and its transfer) between Shigella strains and between Shigella and E. coli strains". Hihon lji Shimpor (in Japanese). 1959(1861):34.

2. Boto L. Horizontal gene transfer in evolution: facts and challenges. Proc Biol Sci. 2010;277(1683):819-27.

3. McDade JE, Shepard CC, Fraser DW, Tsai TR, Redus MA, Dowdle WR. Legionnaires' disease: isolation of a bacterium and demonstration of its role in other respiratory disease. N Engl J Med. 1977;297(22):1197-203.

4. Newton HJ, Ang DK, van Driel IR, Hartland EL. Molecular pathogenesis of infections caused by Legionella pneumophila. Clin Microbiol Rev. 2010;23(2):274-98.

5. Rowbotham TJ. Preliminary report on the pathogenicity of Legionella pneumophila for freshwater and soil amoebae. J Clin Pathol. 1980;33(12):1179-83.

6. Horwitz MA, Silverstein SC. Legionnaires' disease bacterium (Legionella pneumophila) multiples intracellularly in human monocytes. J Clin Invest. 1980;66(3):441-50.

7. Nash TW, Libby DM, Horwitz MA. Interaction between the legionnaires' disease bacterium (Legionella pneumophila) and human alveolar macrophages. Influence of antibody, lymphokines, and hydrocortisone. J Clin Invest. 1984;74(3):771-82.

8. Berger $\mathrm{KH}$, Isberg RR. Two distinct defects in intracellular growth complemented by a single genetic locus in Legionella pneumophila. Mol Microbiol. 1993;7(1):7-19.

9. Marra A, Blander SJ, Horwitz MA, Shuman HA. Identification of a Legionella pneumophila locus required for intracellular multiplication in human macrophages. Proc Natl Acad Sci U S A. 1992;89(20):9607-11.

10. Escoll P, Rolando M, Gomez-Valero L, Buchrieser C. From Amoeba to Macrophages: Exploring the Molecular Mechanisms of Legionella pneumophila Infection in Both Hosts. Curr Top Microbiol Immunol. 2013;376:1-34.

11. Hubber A, Roy CR. Modulation of host cell function by Legionella pneumophila type IV effectors. Annu Rev Cell Dev Biol. 2010;26:261-83.

12. Isberg RR, O'Connor TJ, Heidtman M. The Legionella pneumophila replication vacuole: making a cosy niche inside host cells. Nat Rev Microbiol. 2009;7(1):13-24.

13. Cazalet C, Rusniok C, Bruggemann H, Zidane N, Magnier A, Ma L, et al. Evidence in the Legionella pneumophila genome for exploitation of host cell functions and high genome plasticity. Nat Genet. 2004;36(11):1165-73.

14. Nagai $H$, Kagan JC, Zhu X, Kahn RA, Roy CR. A bacterial guanine nucleotide exchange factor activates ARF on Legionella phagosomes. Science. 2002;295(5555):679-82.

15. Rolando M, Buchrieser C. Post-translational modifications of host proteins by Legionella pneumophila: a sophisticated survival strategy. Future Microbiol. 2012;7(3):369-81.

16. Kubori T, Hyakutake A, Nagai $H$. Legionella translocates an $E 3$ ubiquitin ligase that has multiple U-boxes with distinct functions. Mol Microbiol. 2008;67(6):1307-19.

17. Price CT, Al-Khodor S, Al-Quadan T, Santic M, Habyarimana F, Kalia A, et al. Molecular Mimicry by an F-Box Effector of Legionella pneumophila Hijacks a Conserved Polyubiquitination Machinery within Macrophages and Protozoa. PLoS Pathog. 2009;5(12):e1000704.

18. Ivanov SS, Charron G, Hang HC, Roy CR. Lipidation by the host prenyltransferase machinery facilitates membrane localization of Legionella pneumophila effector proteins. J Biol Chem. 2010;285(45):34686-98.

19. Price CT, Al-Quadan T, Santic M, Jones SC, Abu Kwaik Y. Exploitation of conserved eukaryotic host cell farnesylation machinery by an F-box effector of Legionella pneumophila. J Exp Med. 2010;207(8):1713-26.

20. DebRoy S, Dao J, Soderberg M, Rossier O, Cianciotto NP. Legionella pneumophila type II secretome reveals unique exoproteins and a chitinase that promotes bacterial persistence in the lung. Proc Natl Acad Sci U S A. 2006;103(50):19146-51. 
21. Lifshitz Z, Burstein D, Peeri M, Zusman T, Schwartz K, Shuman HA, et al. Computational modeling and experimental validation of the Legionella and Coxiella virulence-related type-IVB secretion signal. Proc Natl Acad Sci $U$ S A. 2013;110(8):E707-15.

22. Zhu W, Banga S, Tan Y, Zheng C, Stephenson R, Gately J, et al. Comprehensive Identification of Protein Substrates of the Dot/lcm Type IV Transporter of Legionella pneumophila. PLoS One. 2011;6(3):e17638.

23. Crabill E, Schofield WB, Newton HJ, Goodman AL, Roy CR. Dot/lcm-Translocated Proteins Important for Biogenesis of the Coxiella burnetii-Containing Vacuole Identified by Screening of an Effector Mutant Sublibrary. Infect Immun. 2018;86(4).

24. O'Connor TJ, Adepoju Y, Boyd D, Isberg RR. Minimization of the Legionella pneumophila genome reveals chromosomal regions involved in host range expansion. Proc Natl Acad Sci U S A. 2011;108(36):14733-40.

25. Bacigalupe R, Lindsay D, Edwards G, Fitzgerald JR. Population Genomics of Legionella longbeachae and Hidden Complexities of Infection Source Attribution. Emerg Infect Dis. 2017;23(5):750-7.

26. Cazalet C, Gomez-Valero L, Rusniok C, Lomma M, Dervins-Ravault D, Newton HJ, et al. Analysis of the Legionella longbeachae genome and transcriptome uncovers unique strategies to cause Legionnaires' disease. PLoS Genet. 2010;6(2):e1000851.

27. Burstein D, Amaro F, Zusman T, Lifshitz Z, Cohen O, Gilbert JA, et al. Genomic analysis of 38 Legionella species identifies large and diverse effector repertoires. Nat Genet. 2016;48(2):167-75.

28. Gomez-Valero L, Rusniok C, Carson D, Mondino S, Perez-Cobas AE, Rolando M, et al. More than 18,000 effectors in the Legionella genus genome provide multiple, independent combinations for replication in human cells. Proc Natl Acad Sci U S A. 2019:in press.

29. Riedmaier P, Sansom FM, Sofian T, Beddoe T, Schuelein R, Newton HJ, et al. Multiple ecto-nucleoside triphosphate diphosphohydrolases facilitate intracellular replication of Legionella pneumophila. Biochem J. 2014;462(2):279-89.

30. Summers EL, Cumming MH, Oulavallickal T, Roberts NJ, Arcus VL. Structures and kinetics for plant nucleoside triphosphate diphosphohydrolases support a domain motion catalytic mechanism. Protein Sci. 2017;26(8):1627-38.

31. Rolando M, Sanulli S, Rusniok C, Gomez-Valero L, Bertholet C, Sahr T, et al. Legionella pneumophila effector RomA uniquely modifies host chromatin to repress gene expression and promote intracellular bacterial replication. Cell Host Microbe. 2013;13(4):395-405.

32. de Felipe KS, Pampou S, Jovanovic OS, Pericone CD, Ye SF, Kalachikov S, et al. Evidence for acquisition of Legionella type IV secretion substrates via interdomain horizontal gene transfer. J Bacteriol. 2005;187(22):7716-26.

33. Gomez Valero L, Runsiok C, Cazalet C, C B. Comparative and functional genomics of Legionella identified eukaryotic like proteins as key players in host-pathogen interactions. Front Microbiol. 2011;2.

34. Lurie-Weinberger MN, Gomez-Valero L, Merault N, Glockner G, Buchrieser C, Gophna $\mathrm{U}$. The origins of eukaryotic-like proteins in Legionella pneumophila. Int J Med Microbiol. 2010;300(7):470-81.

35. Ensminger AW. Legionella pneumophila, armed to the hilt: justifying the largest arsenal of effectors in the bacterial world. Curr Opin Microbiol. 2016;29:74-80.

36. Escoll $\mathrm{P}$, Mondino S, Rolando M, Buchrieser $\mathrm{C}$. Targeting of host organelles by pathogenic bacteria: a sophisticated subversion strategy. Nat Rev Microbiol. 2016;14(1):5-19.

37. Gomez-Valero L, Rusniok C, Cazalet C, Buchrieser C. Comparative and functional genomics of Legionella identified eukaryotic like proteins as key players in hostpathogen interactions. Front Microbiol. 2011;2:208.

38. Qiu J, Luo ZQ. Legionella and Coxiella effectors: strength in diversity and activity. Nat Rev Microbiol. 2017;15(10):591-605. 
39. Rolando M, Buchrieser C. Legionella pneumophila type IV effectors hijack the transcription and translation machinery of the host cell. Trends Cell Biol. 2014;24(12):771-8.

40. Barlocher K, Welin A, Hilbi H. Formation of the Legionella Replicative Compartment at the Crossroads of Retrograde Trafficking. Frontiers in cellular and infection microbiology. 2017;7:482.

41. Merrill AH, Sandhoff K. Sphingolipids: metabolism and cell signalling In: Vance DE, Vance JE, editors. Biochemistry of Lipids, Lipoproteins and Membranes. 36. Elsevier Science, Amsterdam. ed2002. p. 373-407.

42. Heung JL, Luberto C, Del Poeta M. Role of Sphingolipids in Microbial Pathogenesis. Infect Immun. 2006;74(1):28-39.

43. Shabardina V, Kischka T, Kmita H, Suzuki Y, Makalowski W. Environmental adaptation of Acanthamoeba castellanii and Entamoeba histolytica at genome level as seen by comparative genomic analysis. Int J Biol Sci. 2018;14(3):306-20.

44. Rolando $M$, Escoll $P$, Buchrieser $C$. Legionella pneumophila restrains autophagy by modulating the host's sphingolipid metabolism. Autophagy. 2016;12(6):1053-4.

45. Rolando M, Escoll P, Nora T, Botti J, Boitez V, Bedia C, et al. Legionella pneumophila S1P-lyase targets host sphingolipid metabolism and restrains autophagy. Proc Natl Acad Sci U S A. 2016;113(7):1901-6.

46. Degtyar E, Zusman T, Ehrlich M, Segal G. A Legionella effector acquired from protozoa is involved in sphingolipids metabolism and is targeted to the host cell mitochondria. Cell Microbiol. 2009;11(8):1219-35.

47. Gomez-Valero L, Buchrieser C. Genome dynamics in Legionella: the basis of versatility and adaptation to intracellular replication. Cold Spring Harbor perspectives in medicine. 2013;3(6).

48. Choy A, Dancourt J, Mugo B, O'Connor TJ, Isberg RR, Melia TJ, et al. The Legionella effector RavZ inhibits host autophagy through irreversible Atg8 deconjugation. Science. 2012;338(6110):1072-6.

49. Schmitz-Esser S, Tischler $P$, Arnold R, Montanaro J, Wagner M, Rattei T, et al. The genome of the amoeba symbiont "Candidatus Amoebophilus asiaticus" reveals common mechanisms for host cell interaction among amoeba-associated bacteria. J Bacteriol. 2010;192(4):1045-57.

50. Casadevall A, Fu MS, Guimaraes AJ, Albuquerque P. The 'Amoeboid Predator-Fungal Animal Virulence' Hypothesis. J Fungi (Basel). 2019;5(1).

51. Raoult D, Audic S, Robert C, Abergel C, Renesto P, Ogata H, et al. The 1.2-megabase genome sequence of Mimivirus. Science. 2004;306(5700):1344-50.

52. Moreira D, Brochier-Armanet $C$. Giant viruses, giant chimeras: the multiple evolutionary histories of Mimivirus genes. BMC Evol Biol. 2008;8:12.

53. Ogata H, La Scola B, Audic S, Renesto P, Blanc G, Robert C, et al. Genome sequence of Rickettsia bellii illuminates the role of amoebae in gene exchanges between intracellular pathogens. PLoS Genet. 2006;2(5):e:76.

54. Gomez-Valero L, Neou Bonora M, Gribaldo S, Buchrieser C. Interdomain Horizontal Gene Transfer Shaped the Genomes of Legionella pneumophila and Legionella longbeachae. In: Gophna U, editor. Lateral Gene Transfer in Evolution. New York, NY: Springer; 2013. p. 199-219.

55. Nyvltova E, Sut'ak R, Zarsky V, Harant K, Hrdy I, Tachezy J. Lateral gene transfer of pcresol- and indole-producing enzymes from environmental bacteria to Mastigamoeba balamuthi. Environ Microbiol. 2017;19(3):1091-102.

56. Kaneko T, Stogios PJ, Ruan X, Voss C, Evdokimova E, Skarina T, et al. Identification and characterization of a large family of superbinding bacterial $\mathrm{SH} 2$ domains. Nature communications. 2018;9(1):4549.

57. de Felipe KS, Glover RT, Charpentier X, Anderson OR, Reyes M, Pericone CD, et al. Legionella eukaryotic-like type IV substrates interfere with organelle trafficking. PLoS Pathog. 2008;4(8):e1000117. 
58. Gimenez G, Bertelli C, Moliner C, Robert C, Raoult D, Fournier PE, et al. Insight into cross-talk between intra-amoebal pathogens. BMC Genomics. 2011;12:542.

59. Gomez-Valero L, Rusniok C, Jarraud S, Vacherie B, Rouy Z, Barbe V, et al. Extensive recombination events and horizontal gene transfer shaped the Legionella pneumophila genomes. BMC Genomics. 2011;12:536.

60. Wang Z, Wu M. Comparative Genomic Analysis of Acanthamoeba Endosymbionts Highlights the Role of Amoebae as a "Melting Pot" Shaping the Rickettsiales Evolution. Genome Biol Evol. 2017;9(11):3214-24.

61. Bertelli C, Greub G. Lateral gene exchanges shape the genomes of amoeba-resisting microorganisms. Frontiers in cellular and infection microbiology. 2012;2:110.

62. Buchrieser $\mathrm{C}$, Charpentier $\mathrm{X}$. Induction of competence for natural transformation in Legionella pneumophila and exploitation for mutant construction. Methods Mol Biol. 
A

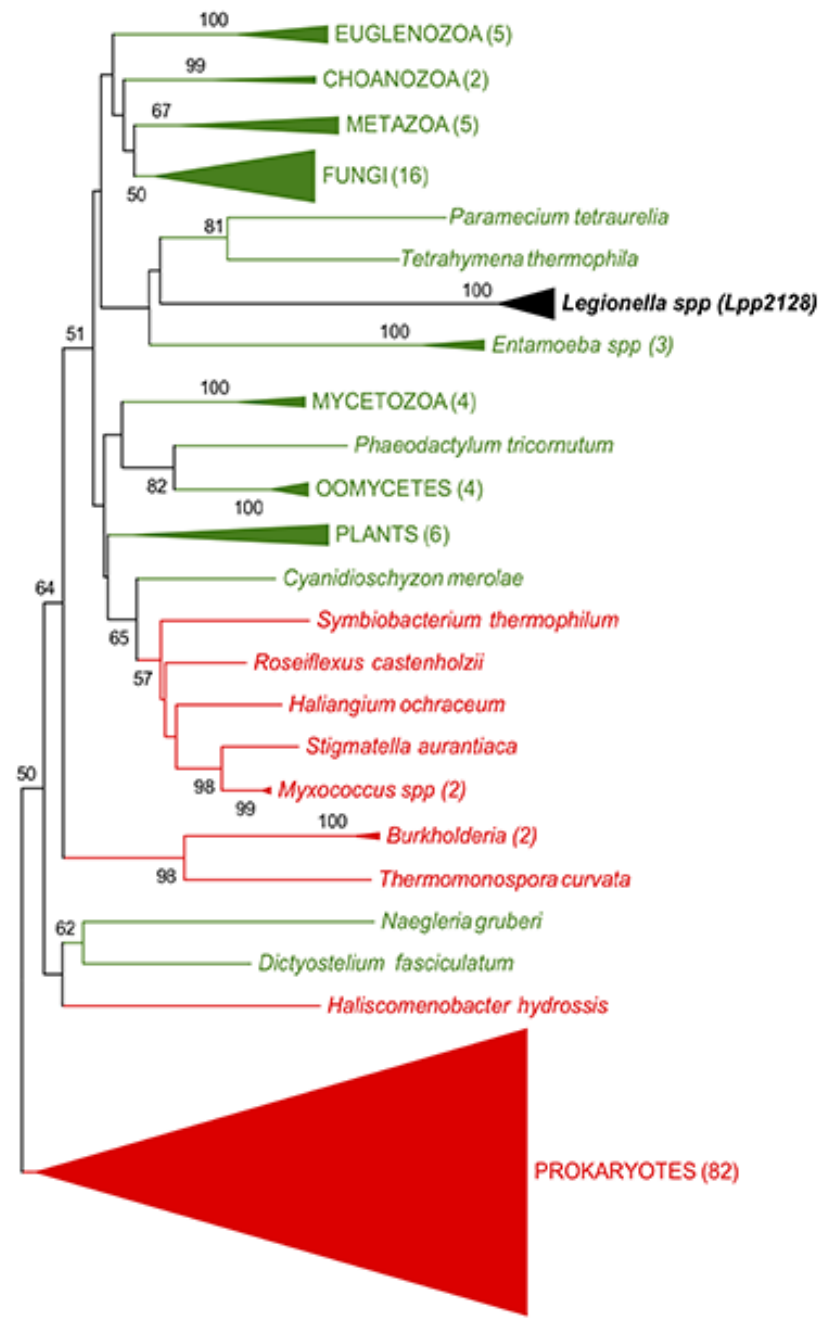

ץ.1
B

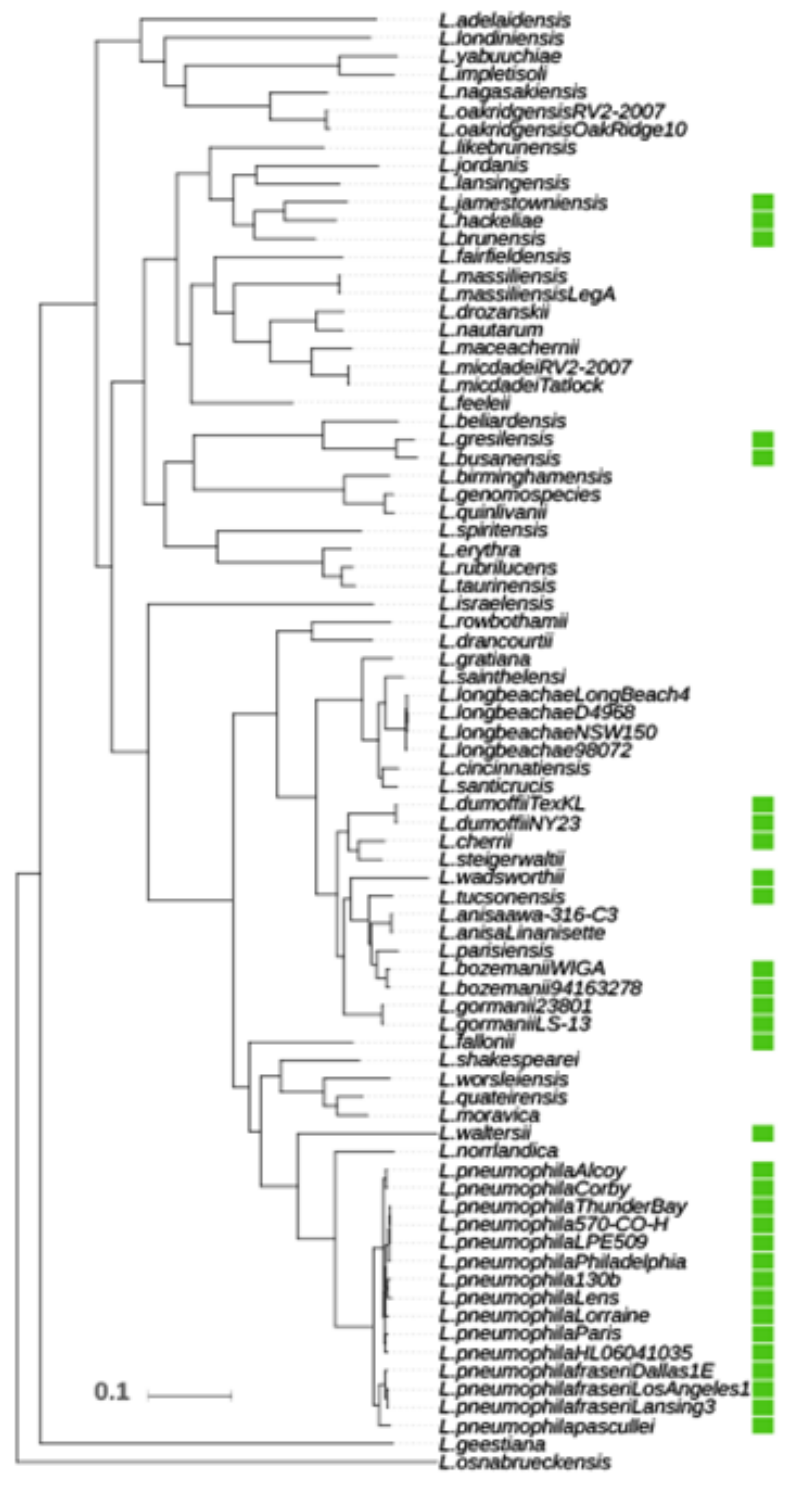




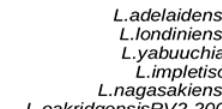

L.impletisol

L.oakridgensisRV2-2007 L.oakridgensisOakRidge10 L.jordanis
Like

L.lansingensis

L.hackeliae
L.hrentonsis L.brunensis

Lassiliensis

L.massiliensis
L.miliensisLegA

L.drozanskii

L.nautarum

L.maceachernii

L. cherrii

L. tucsonensis

$\underbrace{94}_{56} \underbrace{L}_{\text {L. bozemanii } B}$

$63-68 \%$ aa similarity

LicdadelRV2-2007

micdadeiTatlock

L.beliardensis

L.gresilensis

L.busanensis

L.birminghamensis
L.genomospecies

L.quinlivanii

L.spiritensis
Lerythra

L.rubrilucens

L.taurinensis

L.israelensis

L.rowbothamii

L.drancourtii L.gratiana
Lainthelensi

$67-69 \%$ aa similarity L. jamestc $70 \%$ aa similarity $\begin{array}{r}\text { L.IongbeachaeLongBeach4 } \\ \text { L.longbeachaeD4968 }\end{array}$
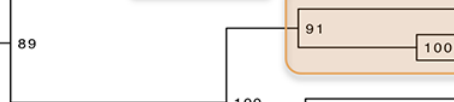
L. hackeliae busanensis L.longbeachaeNSW150 L. longbeachae 98072
L.cincinnatiensis L.santicrucis
L. $L$ pneumophila Philadelphia 2 pneumophila $570 \mathrm{C}$

Loopneumophila LPE509

L. pneumophila Thunderbay

L. L. pneumophila Paris

q. pneumophila HL06041035

${ }^{6}{ }^{6}$, pneumophila Alcoy

59 L. pneumophila Alcoy

$\sigma_{5} \frac{L}{8}$. pneumophila Lens

st. pneumophila $130 \mathrm{~b}$

L. pneumophila Lorraine

- L. pneumophila pascuelleii

${ }_{5} \mathrm{~L}$. pneumophila fraserii $A$

$\frac{7}{45}$ pneumophila fraserii $C$

0.08 1. pneumophila fraserii $C$

\section{$\curvearrowleft$ Gene acquisiton $\bigcirc$ Gene loss}

Tree scale: 0.1

$95-100 \%$

aa similarity

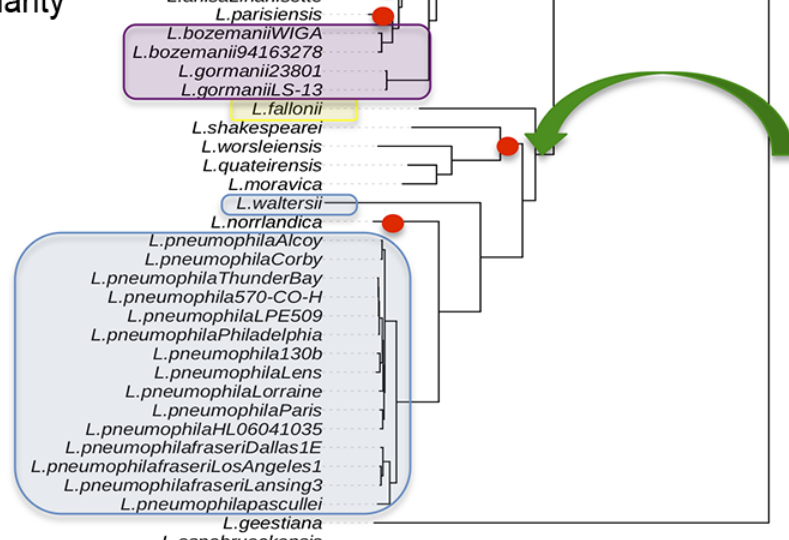

Lisawa-316-c3

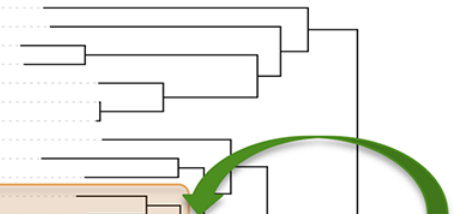

7
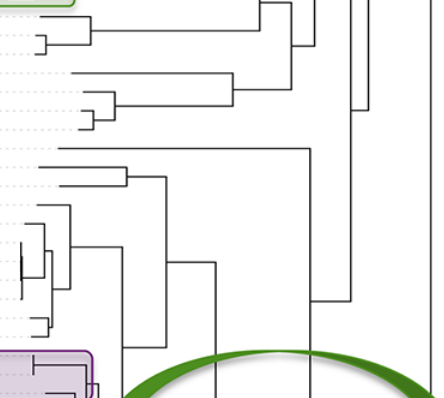
L.dumoffiinY23 L. cherrii
L. dumeigerwatti
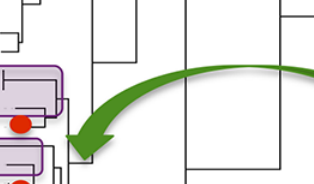

brueckensis 